\title{
ANÁLISIS HIDROGRÁFICO E ICTIOLÓGICO DE LAS CAPTURAS REALIZADAS CON UNA RED DE TRAMPA FIJA EN LA LAGUNA DE GANDOCA, LIMÓN, COSTA RICA
}

\author{
Rosario Benavides Morera ${ }^{1 *}$ y Carlos L. Brenes ${ }^{1}$
}

\begin{abstract}
RESUMEN
Se realizó un estudio ictiológico de las capturas obtenidas con una red de trampa fija y un registro de las propiedades hidrológicas en la Laguna de Gandoca, Limón, entre abril del 2006 y julio del 2007. Se identificaron 13 especies pertenecientes a 10 familias. E1 98\% de las capturas estuvo conformado por cuatro especies de valor comercial: Centropomus pectinatus (77\%), Eucinostomus gula $(9 \%)$, Caranx latus (7\%) y Stellifer colonensis (5\%). C. pectinatus (róbalo) estuvo presente en todas las capturas. Para C. pectinatus, se determinó que la relación longitud total - peso se ajustó potencialmente a través de la ecuación $\mathrm{Pt}=$ $0.004 \mathrm{Lt}^{3.1848}$. La talla de primera madurez de los róbalos se determinó en $30 \mathrm{~cm}$ de longitud total. En el ciclo anual de las propiedades termohalinas superficiales, la temperatura máxima se registró en el mes de setiembre $\left(32^{\circ} \mathrm{C}\right)$ y la mínima durante diciembre $\left(25.5^{\circ} \mathrm{C}\right)$. La laguna exhibe sus mayores salinidades en octubre (21), mientras que las salinidades menores ocurrieron en julio y diciembre (1). Entre setiembre y noviembre, la influencia de la onda mareal se extendió hasta media laguna con salinidades de 20, mientras que en la parte más interna no excedió a 5. Los valores más altos de contenido de oxígeno se observaron entre setiembre y noviembre, cuando el aporte de agua dulce proveniente de las escorrentías es mínimo. Finalmente, las características espacio-temporales del campo salino tienen una influencia directa en la composición y distribución de la ictiofauna que habita en la laguna.
\end{abstract}

Palabras claves: Laguna costera, hidrografía, trampa fija, Gandoca, Costa Rica.

\begin{abstract}
An ichthyological study of the fishing catch in a fixed trap net along with hydrographic sampling of the hydrological properties in Gandoca Lagoon, Limón, was carried out between April 2006 and July 2007. Thirteen species belonging to 10 families were identified. Ninty-eight percent of the captures belong to four species of commercial value: Centropomus pectinatus (77\%), Eucinostomus gula (9\%), Caranx latus (7\%) and Stellifer colonensis (5\%). C. pectinatus was present in all captures. For C. pectinatus (snook), the relationship between total length and weight was adjusted potentially through the equation $\mathrm{Pt}=0.004 \mathrm{Lt}^{3.1848}$. The size of first maturity of snooks was $30 \mathrm{~cm}$. The annual cycle of surface termohaline properties shows the maximum temperature in September $\left(32^{\circ} \mathrm{C}\right)$ and the minimum during December $\left(25.5^{\circ} \mathrm{C}\right)$. The Lagoon exhibits maximum salinities in October (21) and minimum in July and December (1). Between September and November the influence of the tidal wave extends to the middle of the lagoon with salinities of 20, while in the inner part it does not exceed 5. The highest values in oxygen content were observed between September and November, when the contribution of fresh water runoff is minimal. The space-time characteristics of the salinity field have a direct influence on the composition and distribution of the icthyofauna that inhabits the Lagoon.
\end{abstract}

Keywords: Coastal lagoon, hydrography, trap net, Gandoca, Costa Rica.

1 Laboratorio de Oceanografía y Manejo Costero, Universidad Nacional, Heredia, Costa Rica, Apdo. 86-3000. rbmorera@gmail.com*,cbrenes@una.ac.cr

Recibido 25-II-2009

Aceptado 24-VI-2010

DOI: http://dx.doi.org/10.15359/revmar.2.1 


\section{INTRODUCCIÓN}

Las lagunas costeras son cuerpos de agua someros y salobres, separados del ambiente costero por una barrera de arena. Ocupan el $14 \%$ de la zona costera del planeta y sus características más relevantes son la conexión periódica o permanente con el océano y su alta productividad (Yáñez-Arancibia, 1986). En estos ecosistemas, el frágil equilibrio entre el dinámico medio físico y la biota es altamente vulnerable a la acción humana y a los cambios naturales, como inundaciones o intrusiones marinas. Esta compleja variabilidad natural y antrópica debe ser comprendida antes de manejar racionalmente sus recursos (Yáñez-Arancibia, 1986).

La pesca en estos ambientes se ha desarrollado porque en las aguas poco profundas, los ciclos migratorios masivos y relacionados con condiciones estacionales y climáticas han permitido una observación continua y directa por parte del hombre. Esto explica por qué las mismas tecnologías de captura, especialmente las de artes fijas, han tomado siempre el carácter de instrumento de gestión del recurso y de repartición del territorio. Claramente, quien opera con artes de pesca fijas depende de los intercambios entre el mar y la laguna.
En el caso de la captura de adultos y subadultos que migran del mar hacia la laguna, la laguna costera toma carácter de "trampa" y el hombre desarrolla una actividad típica de pesca. En el caso de los peces que han desarrollado sus etapas de crecimiento en estos ambientes costeros, su éxito productivo se basa en las dinámicas tróficas que se generan en el interior del sistema. Esto explica la atención y dedicación por parte del hombre que ha instalado artes fijas de capturas, hacia la hidrodinámica y hacia las posibilidades de intervenir en las bocas para mantener un contacto funcional entre el mar y la laguna. La presencia de artes fijas hace que la laguna adquiera un carácter de área ordenada. De aquí se deriva la posición de quien considera la pesca en lagunas costeras ordenadas con instalaciones fijas de pesca, como lugares donde se desarrolla una verdadera actividad de acuicultura extensiva.

El humedal conocido como Laguna de Gandoca se encuentra ubicado en el Refugio de Vida Silvestre Gandoca-Manzanillo, que es uno de los hábitats más ricos del Caribe de Costa Rica en diversidad biológica $\left(09^{\circ} 35^{\prime} 26^{\prime \prime} \mathrm{N}-82^{\circ} 35^{\prime} 46^{\prime \prime}\right.$, Fig. 1). Sin embargo, es notable la ausen-

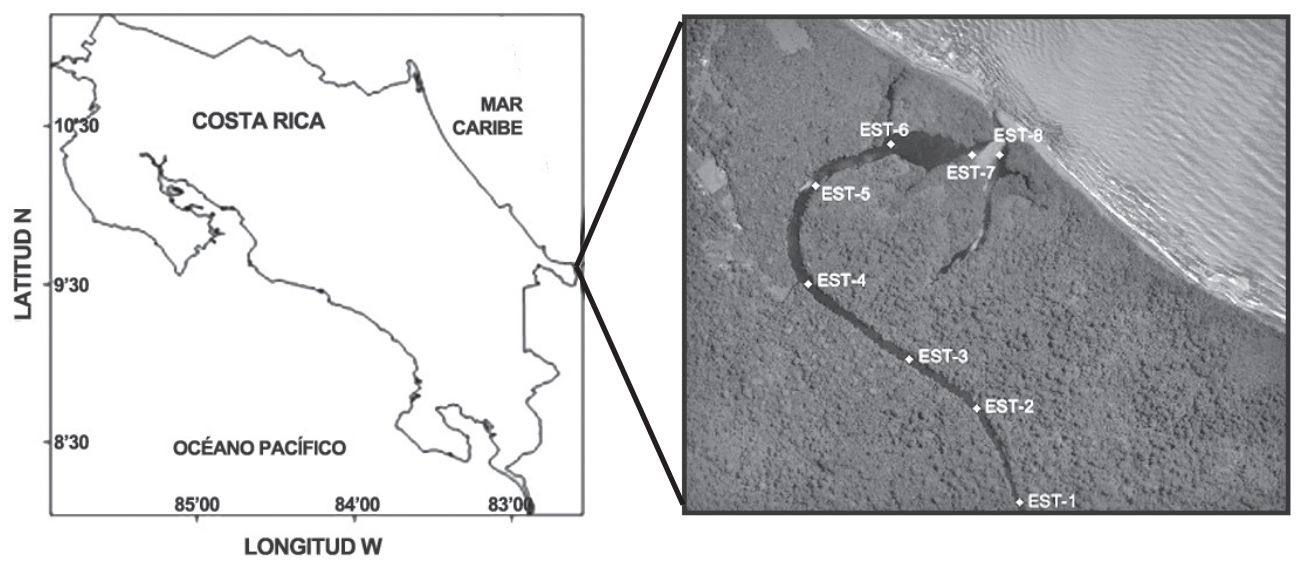

Fig. 1. Posición de las estaciones hidrográficas (adaptado de Google Earth, 2008)

Fig. 1. Location of hydrographic stations (Adapted from Google Earth, 2008) 
cia de investigaciones sistemáticas que permitan conocer y evaluar la riqueza y el potencial del recurso natural en dicha laguna. Windevoxhel et al. (1995) realizaron una caracterización general de la zona, con la finalidad de que el ecosistema fuera incluido dentro de la lista RAMSAR. Coll et al. (2001) determinaron, con técnicas de fotointerpretación, las principales asociaciones vegetales presentes en la laguna. Posteriormente, Harbeyam et al. (2003) realizaron una breve caracterización limnológica del sistema y Gastézzi (2003) hizo un trabajo de evaluación y manejo de sus recursos. Coll et al. (2004) estudiaron la variabilidad estacional de algunas características físicas y químicas de la laguna y la determinación de posibles plaguicidas. Fonseca y Solís (2004) realizaron un trabajo sobre aspectos de manejo conjunto del Refugio Nacional de Vida Silvestre Gandoca-Manzanillo y, finalmente, en el 2006, en el Informe Nacional sobre el Inventario de Cuerpos de Agua Continentales de Costa Rica, realizado por el Proyecto PREPAC (Plan Regional de Pesca y Acuicultura Continental) (2006), se llevó a cabo una descripción general de los aspectos más relevantes de dicho cuerpo de agua.

La Laguna de Gandoca constituye una importante fuente de recursos para el sustento de las familias que habitan en sus alrededores. La comunidad local depende del funcionamiento y productividad de este ecosistema para la obtención de recursos como pesca, leña, madera (construcción), recreación y turismo. Los resultados del presente estudio son de gran utilidad para la comunidad que habita en el entorno de la laguna, por el aporte al conocimiento acerca de la ictiofauna que se encuentra en dicho cuerpo de agua, por los principales aspectos hidrográficos que la gobiernan, por la utilización de un novedoso arte de pesca. Se procura generar información valiosa que pueda ser utilizada como una herramienta de apoyo en la evaluación y manejo de los recursos que conforman este importante ecosistema.

\section{MATERIALES Y MÉTODOS}

La captura de los peces se realizó a través de un arte de pesca fijo denominado "red de almadraba" (Fig. 2). Dicha red es un modelo japonés y el calado o fijación al fondo debe hacerse de forma que se evite el movimiento de las distintas partes que la componen. La almadraba está sostenida firmemente al fondo $(3 \mathrm{~m})$ a travé s de numerosas anclas o pesos muertos y suspendida en la parte superior por boyas. La red se dejó operando 5 días consecutivos en un único sitio cercano a la bocana de la laguna (estación 8, Fig. 1),
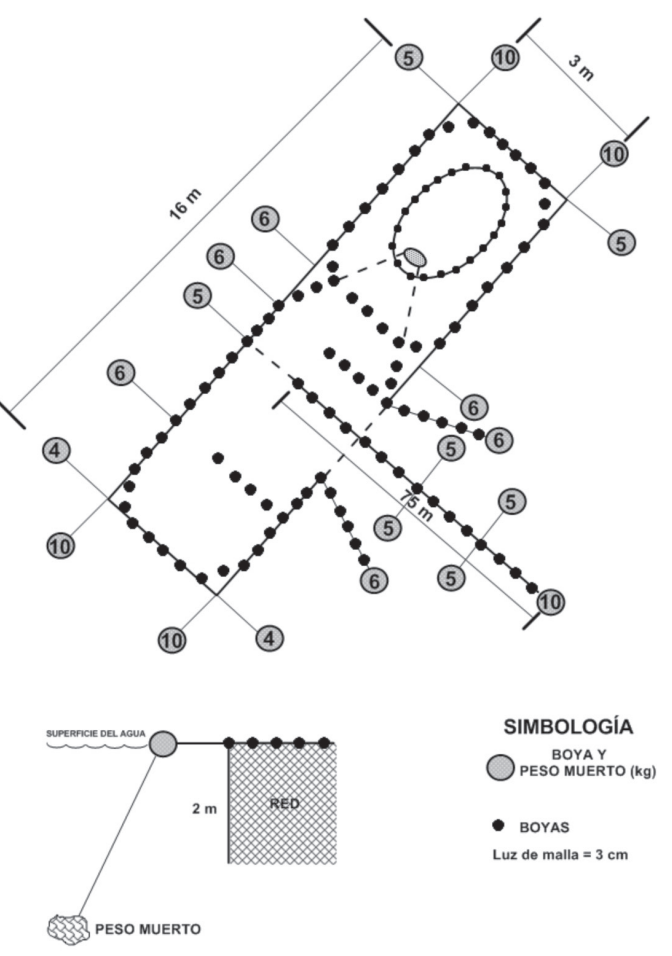

Fig. 2. Diagrama de red de almadraba

Fig. 2. Diagram of "Almadraba" fish trap 
Cuadro 1. Coordenadas geográficas de las estaciones hidrográficas en la Laguna de Gandoca Table 1. Geographic coordinates of the hydrographic stations

\begin{tabular}{cll}
\hline Estación & Latitud N & Longitud O \\
\hline 1 & $09^{\circ} 34^{\prime} 43^{\prime}$, & $82^{\circ} 35^{\prime} 45^{\prime}$, \\
2 & $09^{\circ} 34^{\prime} 55^{\prime}$, & $82^{\circ} 35^{\prime} 52^{\prime}$, \\
3 & $09^{\circ} 35^{\prime} 00^{\prime}$, & $82^{\circ} 36^{\prime} 01^{\prime}$, \\
4 & $09^{\circ} 35^{\prime} 08^{\prime}$, & $82^{\circ} 36^{\prime} 11^{\prime}$, \\
5 & $09^{\circ} 35^{\prime} 20^{\prime}$, & $82^{\circ} 36^{\prime} 10^{\prime}$, \\
6 & $09^{\circ} 35^{\prime} 23^{\prime}$, & $82^{\circ} 36^{\prime} 00^{\prime}$, \\
7 & $09^{\circ} 35^{\prime} 23^{\prime}$, & $82^{\circ} 35^{\prime} 51^{\prime}$, \\
8 & $09^{\circ} 35^{\prime} 25^{\prime}$, & $82^{\circ} 35^{\prime} 47^{\prime}$, \\
\hline
\end{tabular}

con el propósito de aprovechar la dinámica del agua del lugar y las posibles migraciones de la ictiofauna. La recolección de las capturas se realizó mensualmente de abril del 2006 a julio del 2007.

Los peces se identificaron por familia, género y especie, con la ayuda de claves taxonómicas (Perry y Perry, 1974; Bussing, 1998; Cotto, 2001). Se determinó el peso total de los individuos capturados por especie y se registraron los parámetros morfométricos y la biología reproductiva de las especies que representan un interés económico. Se les determinó el largo de horquilla y total (dependiendo de la forma de la aleta caudal de la especie) con un ictiómetro $( \pm 0.5 \mathrm{~cm})$, se les determinó el peso total con una balanza semianalítica $( \pm 0.1 \mathrm{~g})$, se les determinó el sexo y el estadio de desarrollo gonadal por medio del análisis macroscópico de las gónadas, con el fin de limitar las épocas de desove de las especies. El estadio de madurez sexual se estableció de acuerdo con los criterios utilizados por Pérez (1999).

Se realizó un registro mensual de los parámetros hidrológicos en 8 estaciones a lo largo del eje central de la laguna (Fig. 1, Cuadro 1). Se determinó entre la superficie y a $3 \mathrm{~m}$ de profundidad (la profundidad media de la laguna sobre el eje central es de
$4 \mathrm{~m}$ ) la temperatura y el oxígeno disuelto utilizando un oxímetro YSI $55\left( \pm 0.1^{\circ} \mathrm{C} \mathrm{y}\right.$ $\pm 0.01 \mathrm{mg} / \mathrm{L}$, respectivamente) y la salinidad con un refractómetro ( \pm 0.5 PSU). Para la ubicación de las estaciones se utilizó un GPS Garmin, modelo 60.

\section{RESULTADOS}

Las variaciones temporales de la temperatura superficial durante el período de estudio fueron del orden de $\operatorname{los} 4^{\circ} \mathrm{C}$. Una banda de altas temperaturas se observó entre agosto y octubre $\left(\mathrm{T}>31^{\circ} \mathrm{C}\right)$, con dos máximos centrados en setiembre y abril. Las temperaturas más bajas correspondieron a los meses de octubre a enero (Fig. 3A). La estación 4, ubicada muy cerca de un canal secundario poco profundo $(\mathrm{z}<1 \mathrm{~m})$, mostró, de manera general, los valores de temperatura superficial más altos. En la zona interna del sistema se registraron los valores más bajos de temperatura (estaciones 1 y 2), posiblemente debido a la influencia de agua dulce procedente del río Gandoca. Exceptuando los meses comprendidos entre noviembre y enero (cuando los vientos alisios del noreste fortalecen los procesos de mezcla dentro de la laguna), el gradiente espacial de temperatura entre las estaciones 1 y 8 fue del orden de $1^{\circ} \mathrm{C} / \mathrm{km}$. 

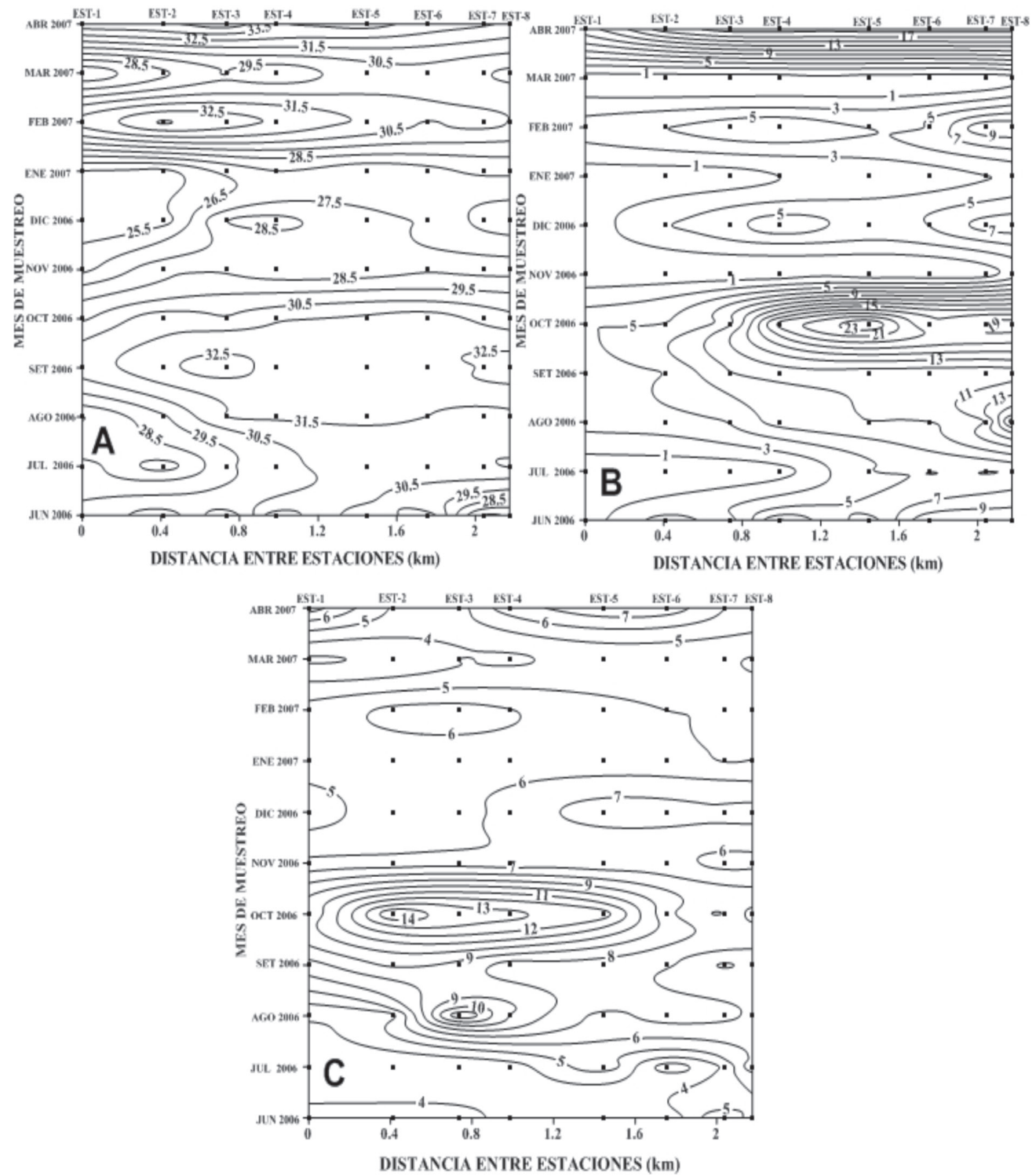

Fig. 3. Variación temporal de la temperatura $\left({ }^{\circ} \mathrm{C}\right)(\mathrm{A})$, salinidad $(\mathrm{B})$ y oxígeno disuelto superficial $(\mathrm{mg} / \mathrm{L})(\mathrm{C})$ en un transepto a lo largo de un eje central en la Laguna de Gandoca

Fig. 3. Temporal variation of surface temperature $\left({ }^{\circ} \mathrm{C}\right)(\mathrm{A})$, salinity $(\mathrm{B})$ and dissolved oxygen $(\mathrm{mg} / \mathrm{L})(\mathrm{C})$ along the central axis in Gandoca Lagoon

Los valores de salinidad presentaron una clara variación temporal, sobre todo en la parte externa del sistema ocupado por las estaciones 5 a 8, con un máximo entre agosto y octubre $(S>5)$, a lo largo de una buena parte de la laguna, incluyendo las áreas más internas (estaciones 2 y 3, Fig. 3B). La parte más externa mantiene un rango de salinidades entre 8 y 24 , mostrando un carácter más oceánico, en concordancia con el trabajo de Coll et al. (2004). 


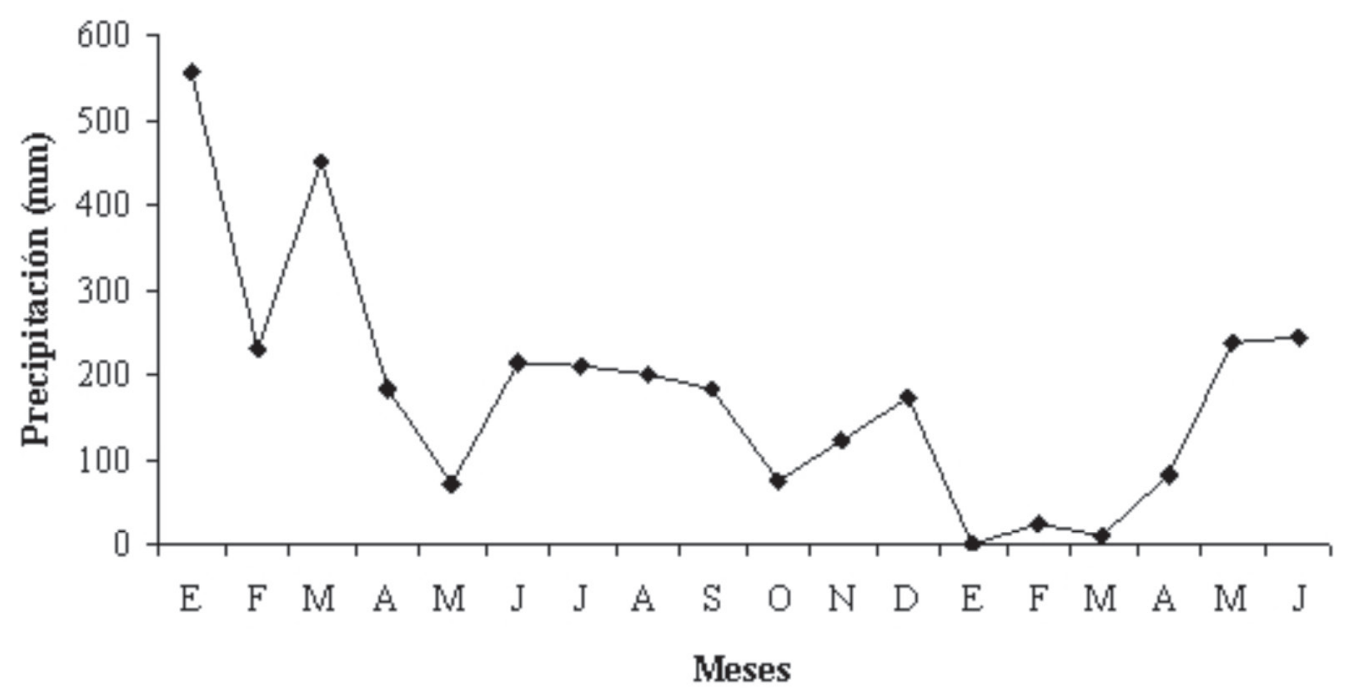

Fig. 4. Precipitación mensual en la estación de Limón para el período 2006-2007 (Instituto Meteorológico Nacional)

Fig. 4. Monthly precipitation at the Limón station for the period 2006-2007 (Instituto Meteorológico Nacional)

La parte externa de la laguna (estaciones 6 a 8) presentó valores relativamente homogéneos de oxígeno disuelto $(\sim 7 \mathrm{mg} / \mathrm{l}$, Fig. 3C). Entre agosto y octubre, meses en los cuales los índices de precipitación en la zona son relativamente bajos (Fig. 4), se registraron valores elevados del contenido de oxígeno que se extendieron desde el interior del sistema.

Se determinaron adicionalmente la temperatura, la salinidad y el oxígeno a $3 \mathrm{~m}$ de profundidad (Fig. 5). Este nivel de la columna de agua se comenzó a determinar a partir de setiembre del 2006. En relación con la temperatura a este nivel, la diferencia térmica con la superficie fue del orden de $1^{\circ} \mathrm{C}$, excepto en enero y diciembre que alcanzó los $2^{\circ} \mathrm{C}$ (Fig. 5A). Un resultado similar fue reportado por Coll et al. (2004). Ambos estratos mostraron, de manera general, una concordancia bastante clara, en cuanto a los períodos en los cuales se observaron las temperaturas más bajas y más altas (Figs. 3A y 5A).
A $3 \mathrm{~m}$ y durante todo el período de muestreo, se evidenció la presencia de una cuña salina, que se extiende desde la parte más externa de la laguna hasta las zonas más internas. Salinidades superiores a 20 se observaron en toda la laguna, excepto para la estación 1, en octubre. El gradiente vertical salino fue del orden de $5 \mathrm{~m}^{-1}$ (Fig. 5B).

Los niveles de oxígeno a $3 \mathrm{~m}$ de profundidad mostraron una disminución importante con respecto a los encontrados en la superficie. El máximo gradiente vertical se obtuvo entre agosto y noviembre (4.5 $\mathrm{mg} / \mathrm{L} / \mathrm{m}$ ), desde la parte central de la laguna hasta el área más interna (entre las estaciones 1 y 4). En general, el oxígeno disuelto a esta profundidad varió entre 1 y $6 \mathrm{mg} / \mathrm{L}$. Los valores más altos se localizaron en las estaciones cercanas a la boca de la laguna, mientras que en las estaciones más alejadas disminuyeron drásticamente (Fig. 5C).

Durante el período de estudio se capturaron en la Laguna de Gandoca un 

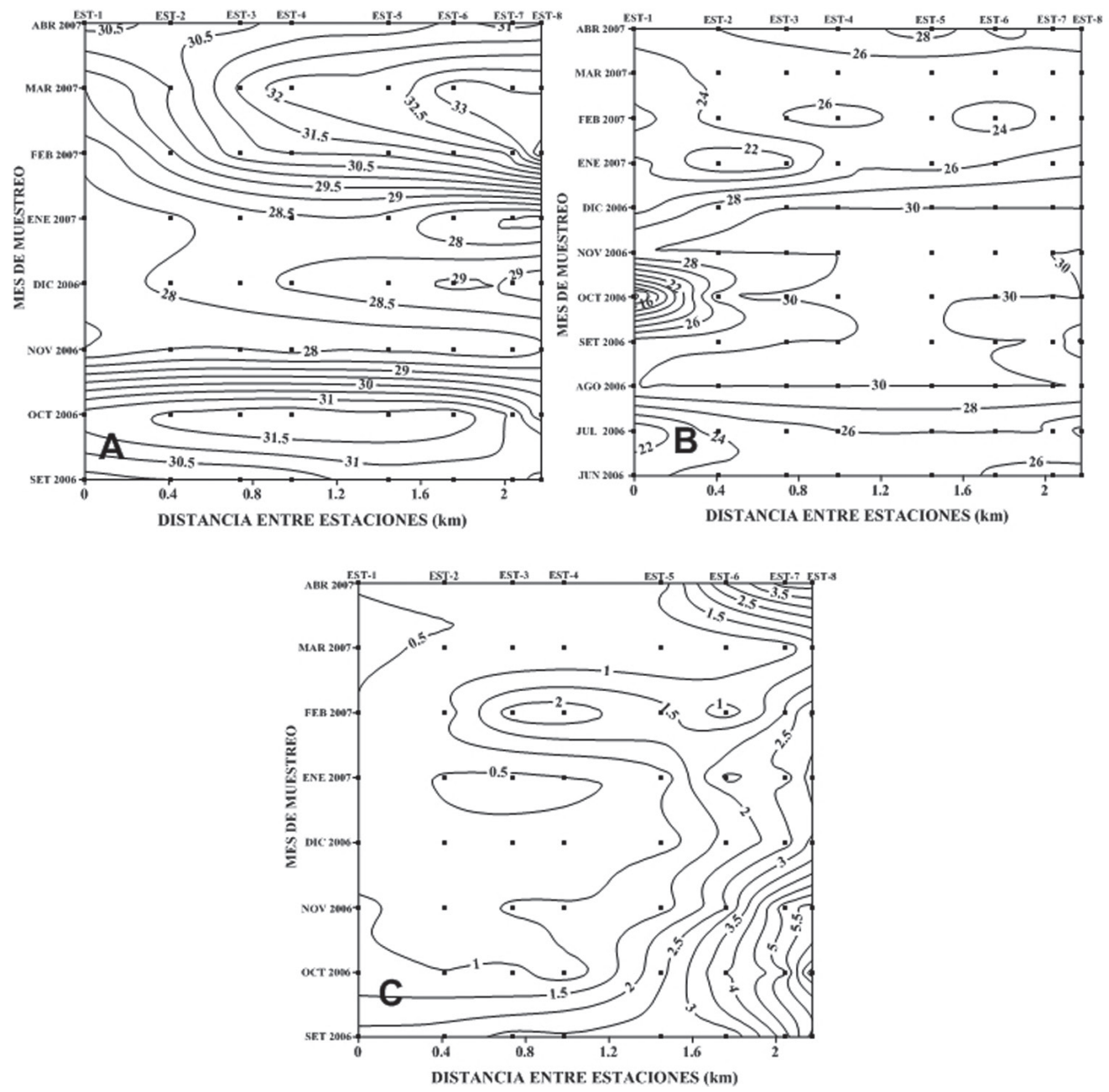

Fig. 5. Variación temporal de la temperatura $\left({ }^{\circ} \mathrm{C}\right)(\mathrm{A})$, salinidad $(\mathrm{B})$ y oxígeno disuelto (C) $(\mathrm{mg} / \mathrm{L})$ a $3 \mathrm{~m}$ en un transepto a lo largo de un eje central en la Laguna de Gandoca

Fig. 5. Temporal variation of temperature $\left({ }^{\circ} \mathrm{C}\right)(\mathrm{A})$, salinity $(\mathrm{B})$ and dissolved oxygen $(\mathrm{mg} / \mathrm{L})(\mathrm{C})$ at $3 \mathrm{~m}$ along the central axis in Gandoca Lagoon

total de 790 individuos, pertenecientes a 10 familias y 13 especies. La familia Centropomidae con una sola especie resultó ser la más numerosa (618 individuos). Le siguieron en importancia, la Gerreidae con 76 especímenes de Eucinostomus gula, la Carangidae queincluyóa 51 ejemplares de tres especies, la Scianidae con 34 individuos de la especie Stellifer colonensis y 6 ejemplares de la familia Lutjanidae. Las restantes cinco familias estuvieron representadas por un único individuo (Cuadro 2).

El cuadro 3 muestra los intervalos de tallas para las especies más abundantes. Centropomus pectinatus es la que presentó el mayor rango de tallas $(10-53 \mathrm{~cm})$. Los ejemplares de menor talla correspondieron a Caranx latus $(18 \mathrm{~cm})$.

Para el análisis de la distribución temporal de las capturas, se tomaron en 
Cuadro 2. Capturas en número de individuos por especie durante el período de muestreo (mayo 2006-julio 2007) en la Laguna de Gandoca

Table 2. Captures in number of individuals by species during the sampling period (May 2006-Julio 2007) in Gandoca Lagoon

\begin{tabular}{llc}
\hline \multicolumn{1}{c}{ Familia } & \multicolumn{1}{c}{ Especie } & Número de individuos \\
\hline Centropomidae & Centropomus pectinatus & 618 \\
Gerreidae & Eucinostomus gula & 76 \\
Carangidae & Caranx latus & 49 \\
& Oligoplites saurus & 1 \\
& Chloroscombus chysurus & 1 \\
Scianidae & Stellifer colonensis & 34 \\
Magalopidae & Megalops atlanticus & 1 \\
Lutjanidae & Lutjanus apodus & 5 \\
& Lutjanus griseus & 1 \\
Clupeidae & Ophistonema oglinum & 1 \\
Paralichtidae & Citharichthys spilopterus & 1 \\
Serranidae & Ephinephelus itajara & 1 \\
Mugilidae & Agonostomus monticula & 1 \\
\hline
\end{tabular}

Cuadro 3. Distribución por rango de tallas de las especies de mayor captura Table 3. Distribution for range of size of the species of more capture

\begin{tabular}{lccc}
\hline Especie & $\begin{array}{c}\text { Rango de tallas } \\
\text { (cm) }\end{array}$ & $\begin{array}{c}\text { Rango de tallas } \\
\text { de mayor captura }(\mathbf{c m})\end{array}$ & $\begin{array}{c}\text { Número de } \\
\text { individuos }\end{array}$ \\
\hline C. pectinatus & $10-53$ & $10-20$ & 281 \\
E. gula & $9-40$ & $9-20$ & 58 \\
C. latus & $9-18$ & $12-15$ & 20 \\
S. colonensis & $7-27$ & $15-20$ & 24 \\
\hline
\end{tabular}

cuenta las cuatro especies que mostraron la mayor abundancia y relevancia económica (Fig. 6). Las mayores abundancias de $C$. pectinatus se registraron en agosto y abril (Fig. 6A), mientras que para E. gula (Fig. 6B), C. latus (Fig. 6C) y S. colonensis (Fig. 6D) se obtuvieron los niveles más altos de su captura en junio. Es notable que para C. pectinatus se lograran obtener capturas en todos los meses del año, mientras que para las demás especies hubo ausencia de individuos en al menos tres meses.
C. pectinatus presentó poca variación de su talla promedio $(30 \mathrm{~cm})$ a todo 10 largo del año (Fig. 7A); para E. gula, en los meses de octubre, marzo y julio se registraron los individuos de mayor tamaño (Fig. 7B). C. latus se capturó en tamaños mayores durante los meses de junio, agosto y octubre (Fig. 7C) y S. colonensis, en diciembre (Fig. 7D).

Con respecto a la proporción por sexos, en $C$. pectinatus se observó que los machos $(30 \%)$ duplicaron a las hembras (15\%). 

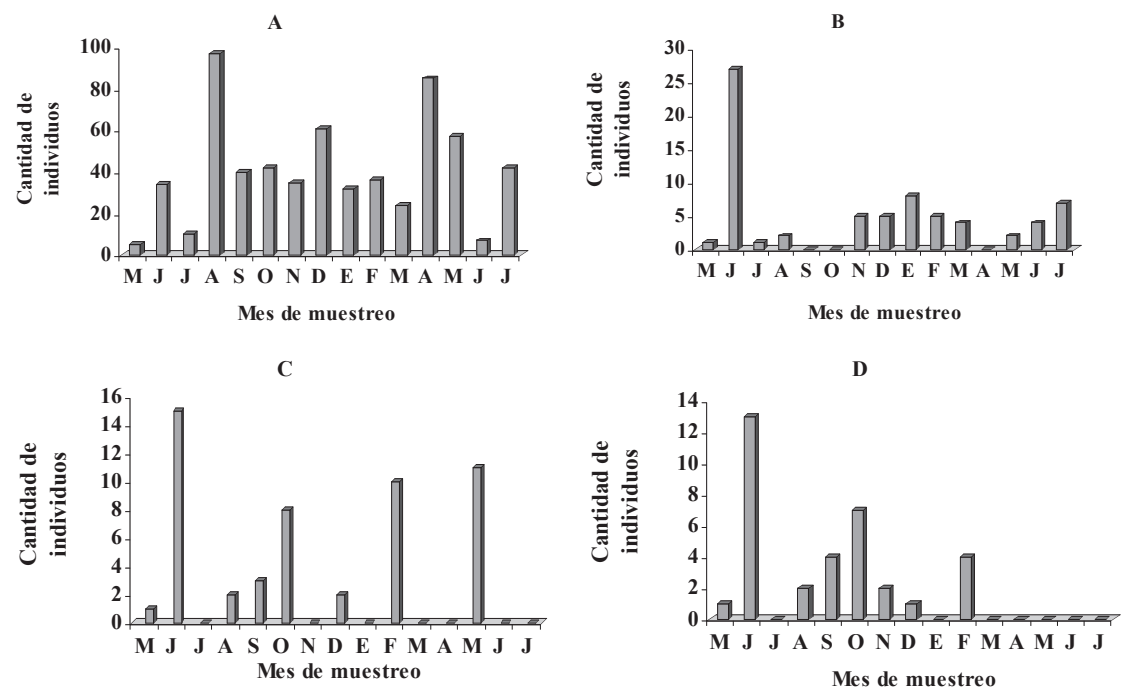

Fig. 6. Distribución temporal de las capturas para las cuatro especies más abundantes: $C$. pectinatus (A), E. gula (B), C. latus (C), S. colonensis (D)

Fig. 6. Temporal distribution of captures for the most abundant species C. pectinatus (A), E. gula (B), C. latus (C), S. colonensis (D)

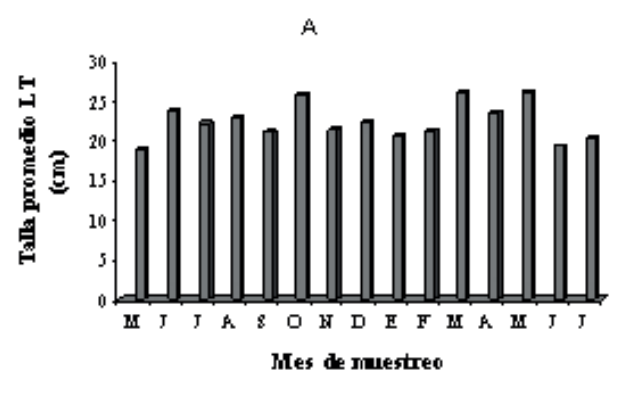

C

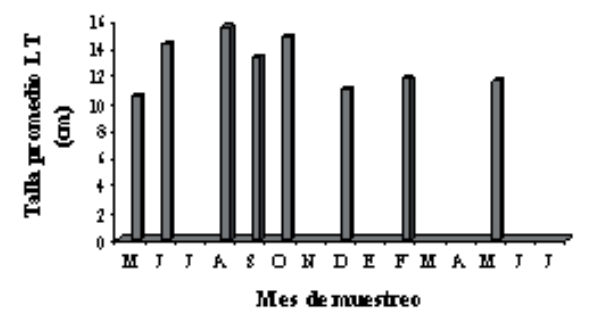

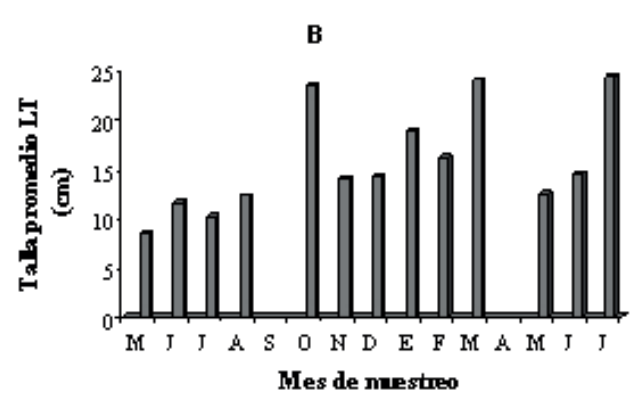

D

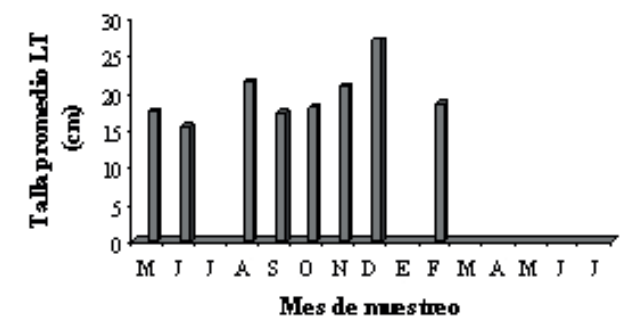

Fig. 7. Distribución temporal de frecuencias de tallas para C. pectinatus (A), E. gula (B), C. latus (C), S. colonensis (D)

Fig. 7. Temporal distribution of size frequencies of C. pectinatus (A), E. gula (B), C. latus (C), S. colonensis (D) 


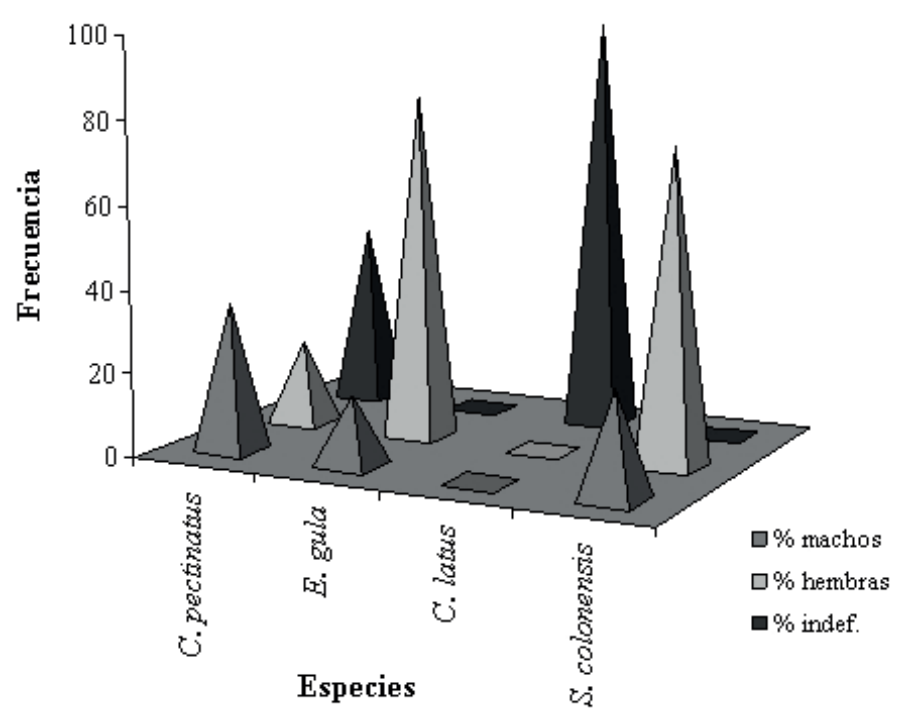

Fig. 8. Proporción de sexos de las especies de mayor abundancia durante el período de estudio Fig. 8. Sex ratios for the most abundant species during the period of study
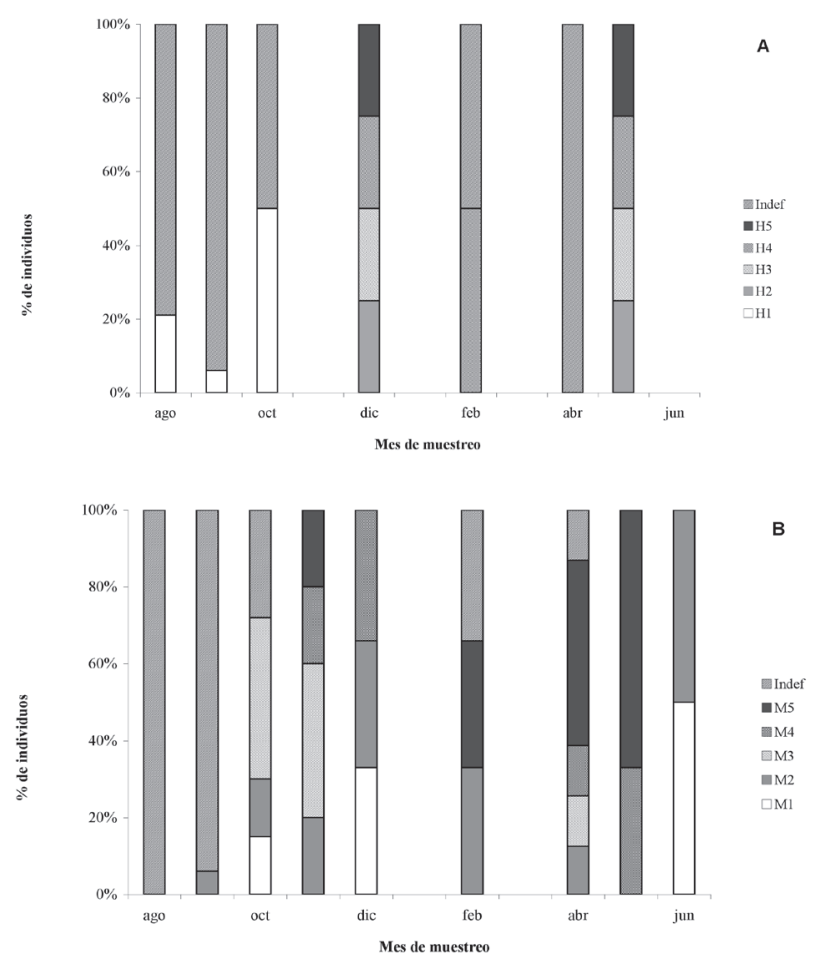

Fig. 9. Proporción de los estadios de madurez sexual de C. pectinatus hembras (A) y machos (B), durante el período de estudio

Fig. 9. Proportion of sexual maturity stages of $C$. pectinatus females (A) and males (B), during the period of study 


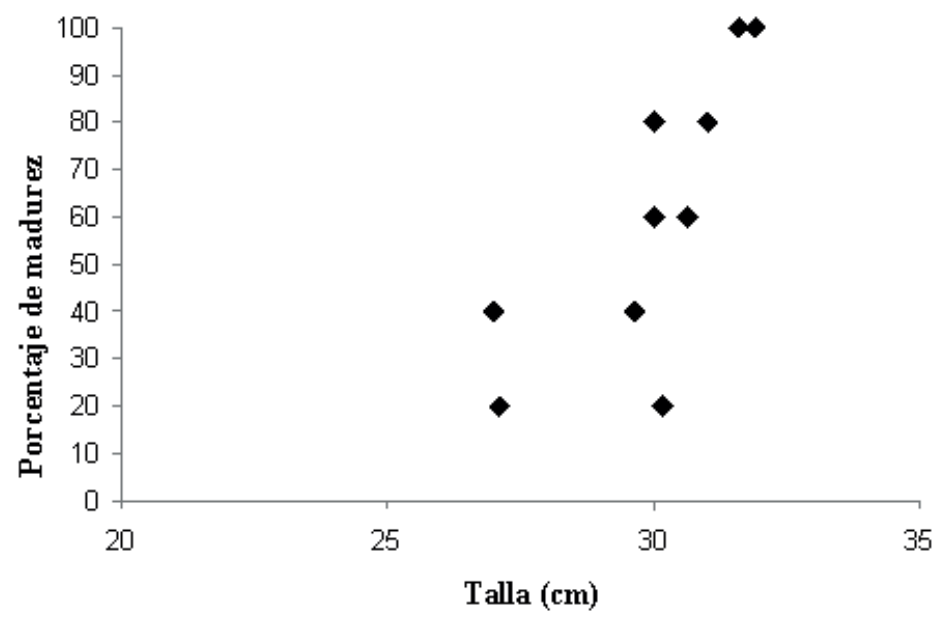

Fig. 10. Porcentaje de madurez con respecto a la talla en C. pectinatus

Fig. 10. Percentage of maturity relative to size in C. pectinatus

Por el contrario, para E. gula, alrededor del $80 \%$ de la población fueron hembras, mientras que los machos conformaron casi el 20\%. Todos los especímenes capturados de la especie C. latus fueron indefinidos y, finalmente, para S. colonensis, el mayor porcentaje estuvo representado por las hembras (70\%) (Fig. 8).

En relación con la maduración sexual, el mayor porcentaje de hembras de C. pectinatus presentó en agosto los diferentes estadios de maduración gonadal (Fig. 9). Para las hembras, en los primeros meses de muestreo el mayor porcentaje eran sexualmente indefinidas; en diciembre y mayo mostraban algún grado de madurez (estadio II, III, IV y V, cada uno con un $25 \%$ de las hembras maduras). La maduración sexual en los machos comienza a observarse a partir de octubre del 2006 y en el mes de noviembre, el 20\% está en estadio II, el $40 \%$ en estadio III, el $20 \%$ en estadio IV y el $20 \%$ en estadio V. En los meses de abril y mayo del 2007, los individuos están potencialmente maduros en un $50 \%$ y $70 \%$, respectivamente.
En la figura 10 se muestra la talla promedio a la que los róbalos (C. pectinatus) alcanzan la madurez sexual. Se observa que en la talla de $30 \mathrm{~cm}$ se ubican los mayores porcentajes de individuos maduros.

El análisis de las relaciones morfométricas se realizó solamente para róbalos, por ser los más abundantes en las capturas totales obtenidas. La figura 11 muestra la relación de naturaleza potencial entre el largo total (Lt) y el peso total $(\mathrm{Pt})$ para la población capturada de $C$. pectinatus, con un $R^{2}=0.977$. Esta relación describe el crecimiento relativo en peso y permite estudiar las variaciones espacio-temporales en el factor de condición fisiológica.

\section{DISCUSIÓN}

En un ambiente lagunar de carácter tropical como la Laguna de Gandoca, las temperaturas superficiales son altas y presentan variaciones temporales relativamente pequeñas.

La Laguna de Gandoca se localiza en una zona donde el régimen de precipitación 


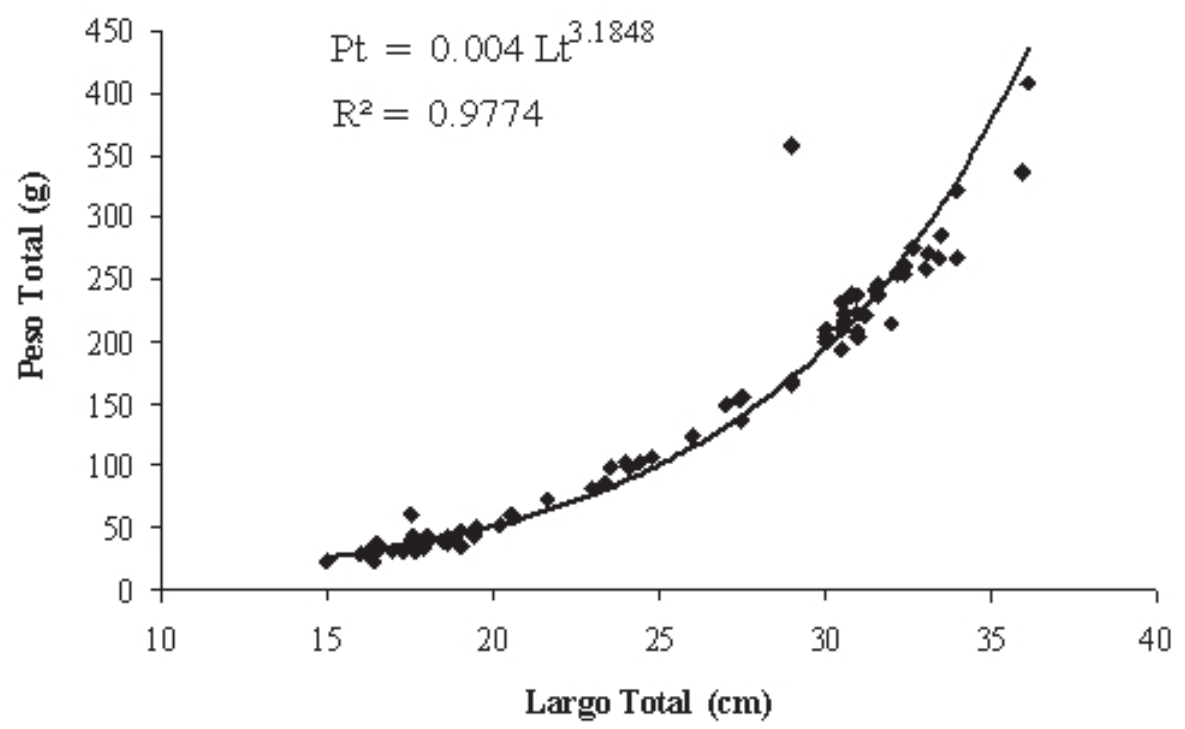

Fig. 11. Largo total (cm) vs. peso total (g) de C. pectinatus

Fig. 11. Total length $(\mathrm{cm})$ vs. total weight $(\mathrm{g})$ in C. pectinatus

anual varía entre los 0-600 $\mathrm{mm}$. Las 1luvias están presentes prácticamente durante los 12 meses, siendo menos intensas entre agosto y octubre. Durante el año 2006, la región Caribe (particularmente la parte costera) registró escenarios entre normales y secos desde abril. El mes más seco fue octubre, el cual se clasificó dentro del escenario seco extremo. El primer trimestre de dicho año fue más lluvioso de lo normal, llegando, inclusive, a superar el escenario lluvioso extremo en marzo (Fig. 4) (Instituto Meteorológico Nacional, 2007). Este comportamiento de las precipitaciones incidió directamente en el campo salino superficial, el cual, contrario a lo observado con la temperatura, muestra un ciclo estacional muy marcado. Esto se debe a que los procesos de dilución de la laguna están muy influenciados por la descarga de agua dulce proveniente principalmente del río Gandoca. No se han registrado otros cauces de agua que lleguen a la laguna. Sin embargo, cuando el régi- men de precipitaciones es muy elevado, la laguna se nutre del agua de escorrentía del Valle Sixaola. Estos aportes, a su vez, presentan una variación temporal significativa en sus caudales, asociada al ciclo anual de lluvias en esta zona (Coll et al. 2004).

En junio y julio, las precipitaciones en la zona son significativas y, en consecuencia, también lo es el aporte de agua dulce por escorrentía. Durante estos meses, las salinidades en toda la laguna son inferiores a 10. La forma de las isohalinas evidencia un predominio del agua dulce sobre el agua salada transportada por la onda mareal. En estas circunstancias, la energía requerida por los procesos de mezcla en la laguna es aportada por el flujo del río.

La cuña salina en los niveles más profundos de la laguna ( $>3 \mathrm{~m}$ ) no se mezcla en su totalidad con el agua menos densa de las capas superficiales, de modo que la laguna se comporta como un estuario fuertemente estratificado (Tipo A) o parcialmente 
mezclado (Tipo B), según la clasificación de Dyer (1979) y Pickard (1990).

A partir de junio (la época lluviosa está claramente definida), las aguas superficiales de la laguna muestran una importante disminución en su contenido de oxígeno $(<4$ $\mathrm{mg} / \mathrm{L}$ ). En este período se presenta un aumento de la carga de materia orgánica disuelta y suspendida que proviene del aporte de los ríos y la cual es degradada. Durante estos meses de mayor precipitación anual, con la disminución de la salinidad se mantiene en solución buena parte del oxígeno procedente de los ríos. El volumen de agua procedente del río no solo transporta materia orgánica particulada, sino que, dada su poca profundidad, contribuye al proceso de remoción de sedimentos del fondo (Coll et al. 2004).

Muestreos previos lograron determinar en la laguna la presencia de 10 especies (Zúñiga, 2005), de las cuales 4 se identificaron en el presente estudio. Los individuos más frecuentes fueron los pertenecientes a las familias: Centropomidae, Lutjanidae, Mugilidae y Gerreidae, todas ellas con especies que habitan en las lagunas costeras en México (Castro-Aguirre et al. 1999) y Nicaragua (Cotto, 2001).

El arte de pesca utilizado durante el muestreo y el período de duración en el agua pueden ser dos de los factores determinantes de las características de las capturas. En general, la red usada es un prototipo de una red que normalmente posee grandes dimensiones $(300 \mathrm{~m} \times 50 \mathrm{~m}$, con una longitud de cola que puede alcanzar varios kilómetros) y ha sido utilizada desde sus inicios para la pesca principalmente de atún en mar abierto, donde es mantenida en el área de pesca y solo se le da mantenimiento periódico. En nuestro caso, por tratarse de un refugio de vida silvestre, la duración de la red en el agua no podía extenderse por un período muy prolongado.

En la distribución temporal de las capturas por especie, las mayores abundancias de C. pectinatus se obtuvieron de agosto del 2006 a mayo del 2007. Trabajos previos con esta especie en ambientes lagunares del Caribe muestran que sus mayores abundancias ocurren durante el segundo semestre del año (Pérez, 1999). En los meses de abril a julio del 2006 se obtuvieron capturas muy diferentes a las del 2007 para esos mismos meses, probablemente a causa de una variación en los rangos de salinidad entre ambos períodos, debido a un cambio interanual considerable observado en los índices de precipitación en dichos meses (Fig. 4).

Las capturas de $S$. colonensis fueron más persistentes en la segunda mitad del año (de agosto a diciembre del 2006). En los casos de E. gula y C. latus no se observó un patrón temporal definido en sus capturas.

La salinidad promedio entre la superficie y a $3 \mathrm{~m}$ de profundidad presentó una correlación positiva $\left(R^{2}=0.53\right)$ y significativa, de acuerdo con un análisis de varianza de una vía $(P=0.002)$, con el número de individuos capturados (Fig. 12). Esto sugiere que existe una relación entre la fluctuación estacional de la salinidad y la abundancia del róbalo, C. pectinatus. Las mayores capturas fueron obtenidas cuando la laguna mostraba las salinidades más altas durante la época seca. Los róbalos son especies demersales que habitan fondos blandos, arenosos y fundamentalmente fangosos de aguas no muy profundas. En etapas tempranas de crecimiento, se asocian a comunidades de manglares, de lirios acuáticos o pastos marinos. De acuerdo con su ciclo biológico, alternan su permanencia en aguas marinas costeras, esteros, ríos y lagunas, 


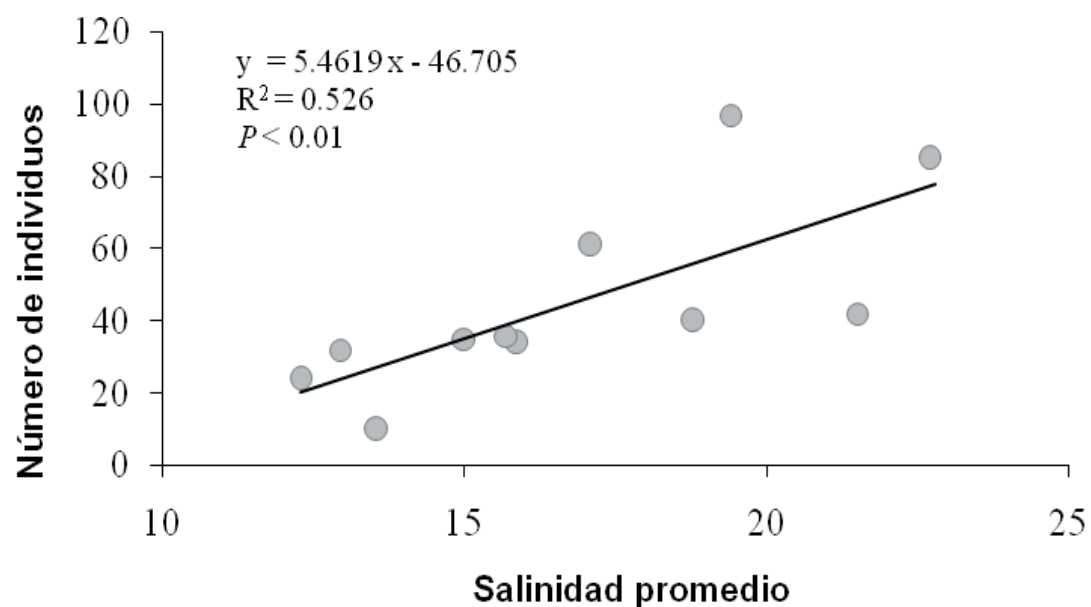

Fig. 12. Salinidad promedio (superficie, $3 \mathrm{~m}$ ) vs. el número de individuos de C. pectinatus capturados Fig. 12. Average salinity (surface, $3 \mathrm{~m}$ ) vs. number of individuals of $C$. pectinatus captured

mostrando así su amplia tolerancia a la concentración salina.

Se desconocen con exactitud los límites de su rango de tolerancia a los cambios de temperatura. Sin embargo, el hecho de habitar aguas poco profundas sugiere que soportan fluctuaciones térmicas considerables. Según Howells y Sunski (1990), esta especie tolera temperaturas entre $\operatorname{los} 28^{\circ} \mathrm{C}$ y $35^{\circ} \mathrm{C}$. Son consumidores terciarios, los adultos son carnívoros muy voraces, se alimentan de numerosas especies juveniles de peces como anchoveta, mojarra, ronco, bagre, guabina, corvina y sardinas, entre otros; de crustáceos como camarones y jaibas; de moluscos como caracoles y almejas. En la etapa juvenil se alimentan de zooplancton (Quiroga et al. 1996).

Las hembras maduras desovan en la cercanía de la costa, y en las etapas de larva y prejuveniles comienzan a emigrar hacia las lagunas costeras y esteros, aprovechando la cuña salina que penetra con la marea. Las lagunas costeras o ríos son utilizados para crecer hasta alcanzar la madurez sexual. En el período próximo al desove efectúan, de nuevo, una migración reproductora hacia la desembocadura de las lagunas costeras hasta alcanzar la franja litoral para desovar (Green, 1993).

En general, los róbalos (C. pectinatus) fueron los peces capturados de mayor talla promedio, fluctuando entre los 18 y $26 \mathrm{~cm}$ (Fig. 7A). Para esta especie, la distribución fue bastante homogénea, aunque los especímenes de mayor talla se capturaron entre marzo y mayo del 2007. Este comportamiento en la distribución de tallas de róbalo coincide con los resultados obtenidos por Pérez (1999) en la laguna de Perlas, en el Caribe de Nicaragua. Para E. gula (mojarra), las tallas mayores se encontraron entre octubre del 2006 y enero y abril del 2007. En los casos de las otras dos especies (C. latus (jurel) y S. colonensis (corvina)), hay una tendencia a encontrar tallas mayores durante el segundo semestre del año. El mismo autor observó que en el caso de la mojarra existe una tendencia a que la talla promedio aumente a través del año, coincidiendo con los resultados obtenidos 
en el presente trabajo (Fig. 7B). Para los jureles, las tallas más grandes tienden a decrecer después de medio año (Fig. 7C), coincidiendo nuevamente con lo encontrado por Pérez (1999). Finalmente, en el caso de la corvina, la distribución de tallas fue bastante homogénea (Fig. 7D).

En el análisis de la proporción de sexos por especie, en $C$. pectinatus se observó un predominio de machos sobre las hembras (1.7:1), mientras que lo contrario se presenta en E. gula (0.2:1) y en $S$. colonensis $(0.3: 1)$ (Fig. 8). La mayor cantidad de especímenes indefinidos se observaron en C. latus, debido probablemente, a que todos los individuos capturados presentaron tallas mucho menores que la talla de primera madurez sexual estimada en $40 \mathrm{~cm}$ (Pérez, 1999). Todos los ejemplares de $C$. latus estuvieron comprendidos en la categoría de indefinidos. Por el contrario, Castro (2000) registró con róbalos (Centropomus parallelus), en la Barra del Colorado, una dominancia de hembras (2.2:1). La proporción en que los individuos de diferentes sexos se encuentran en una población es el resultado de connotaciones adaptativas relacionadas con la reproducción. Sin embargo, este valor no es absoluto, debido a que la proporción sexual está sujeta generalmente a los cambios en el hábitat de cada sexo y este, a su vez, puede cambiar durante cada ciclo anual (García et al. 1994).

Para el caso de C. pectinatus, entre octubre del 2006 y febrero del 2007 se registraron, para ambos sexos, individuos en algún estadio de maduración. En el caso particular de los machos, ese período se extiende hasta julio del 2007. En diciembre (2006), el 100\% de las hembras y los machos capturados presentaron algún grado de maduración en sus gónadas, relacionadas estas con consecuentes períodos de desove (Fig. 8).
En el caso de los machos, en el mes de agosto no hubo actividad de maduración sexual, a partir de setiembre y octubre, las gónadas masculinas presentaron señales de madurez, siendo noviembre, febrero, abril y mayo los meses donde estaban sexualmente maduras. El comportamiento sexual de los róbalos, en general, está marcado por un claro período de desove. Los resultados del presente trabajo podrían indicar que este período es entre noviembre y mayo.

Con respecto al porcentaje de madurez en relación con la talla, para $C$. pectinatus se observó la presencia de gónadas maduras desde los 30 hasta los $32 \mathrm{~cm}$, tanto para hembras como para machos, $\mathrm{y}$ la talla de primera madurez a los $30 \mathrm{~cm}$ (Fig. 10). Yáñez-Arancibia (1977) reporta para $C$. pectinatus la talla de primera madurez entre 29.7 y $31 \mathrm{~cm}$ para hembras y $26.1 \mathrm{~cm}$ para machos, lo que coincide con nuestros resultados.

Finalmente, mediante la ecuación $\mathrm{Pt}=$ $\mathrm{a} * \mathrm{Lt}^{\mathrm{b}}$ fue posible determinar que el crecimiento observado para C. pectinatus fue de tipo isométrico. Un valor de b cercano a 3 indica un crecimiento isométrico, y cuando se aleja de 3 es alométrico (Grimes, 1987; Ruiz et al. 1997). El valor de $b=3.1848$ para nuestro caso (Fig. 11) es indicativo de un crecimiento relativo en peso isométrico. Valores cercanos al anterior fueron reportados para Centropomus undecimalis (3.02) y C. parallelus (3.13) (Zarza et al. 2006).

Dicha relación muestra que la ganancia que tiene el organismo en peso es proporcional a la ganancia que tiene en longitud. Las diferencias o similitudes con respecto al valor de "b", además de ser atribuidas a factores intrínsecos (genéticos) de las especies, se relacionan también con el hábitat y con la época del año. Álvarez (1997) y Marcano (1990) coinciden en este aspecto y mencio- 
nan el estado de explotación de los recursos como otra posible causa de variación.

\section{CONCLUSIONES}

La distribución espacio-temporal de las propiedades termohalinas en la laguna está gobernada por la influencia directa de la marea y del régimen de precipitación que impera en el área. Las mayores salinidades ocurren en octubre, y las menores en julio y diciembre, cuando los índices de precipitación anual sobre la zona estudiada alcanzan también sus máximos estacionales. En época lluviosa, la isohalina de uno se extiende en algunos casos a través de prácticamente todo el ecosistema, y la laguna presenta durante este período un carácter sobre todo dulce-acuícola. Los valores más bajos de temperatura ocurren entre noviembre y enero. Para el caso del oxígeno disuelto, los valores más altos se observaron entre setiembre y noviembre, cuando el aporte de agua dulce proveniente de las escorrentías es mínimo. En la época lluviosa durante el segundo semestre del año, las aguas de la laguna muestran una importante disminución en su contenido de oxígeno, debida principalmente a la degradación de materia orgánica proveniente de la descarga de los ríos.

Térmicamente, la laguna no mostró una estratificación significativa entre la superficie y el fondo, con un valor máximo de $0.6^{\circ} \mathrm{C} / \mathrm{m}$ en el mes de diciembre. En los casos de la salinidad y el oxígeno disuelto, dichos gradientes fueron más notables con valores de $5 \mathrm{~m}^{-1}$ y $4.5 \mathrm{mg} / \mathrm{L} / \mathrm{m}$, respectivamente.

La "red de almadraba" utilizada durante el presente estudio mostró ser un arte de pesca fijo eficiente dentro de un ambiente lagunar. De las especies capturadas en el presente trabajo, tres no han sido reportadas en trabajos previos ( $S$. colonensis, Citharichthys spilopterus y Epinephelus itajara). C. pectinatus fue la única especie presente durante todos los muestreos, y al igual que E. gula y C. latus, las mayores capturas se asociaron a los meses con menos precipitaciones. Se estimó que la talla de primera madurez de los róbalos es de 30 $\mathrm{cm}$. La salinidad, contrario a la temperatura, fue el factor que mostró una influencia directa en la composición y distribución de la ictiofauna que habita en la laguna.

\section{AGRADECIMIENTOS}

Este trabajo se realizó gracias al apoyo brindado por la Universidad Nacional, a través del Proyecto de Investigación SERIO (Servicio Regional e Información Oceanográfica), adscrito al Laboratorio de Oceanografía y Manejo Costero del Departamento de Física y al Instituto Internacional del Océano (IOI).

\section{BIBLIOGRAFÍA}

Álvarez, R. (1997). Explotación mediante el sistema de arrastre y algunos aspectos biológicos del roncador Micropogonias furnieri (Desmarest, 1823) (Pisces: Sciaenidae) en el Golfo de Venezuela. Tesis de Maestría no publicada. Instituto Oceanográfico de Venezuela.

Bussing, W. A. (1998). Peces de las aguas continentales de Costa Rica. San José, Costa Rica: Editorial de la Universidad de Costa Rica.

Castro-Aguirre, J. L., Espinoza-Pérez, H. S. \& Schmitter-Soto, J. J. (1999). Ictiofauna estuarino-lagunar y vicaria de México. Noriga Instituto Politécnico Nacional. México, D.F.: Editorial LIMUSA. 
Castro, M. C. (2000). Aspectos de la biología y estructura poblacional de Centropomus parallelus (Poey, 1860) durante el período de desove en barra del Colorado, Limón, Costa Rica. Tesis de Licenciatura no publicada. Universidad Nacional, Heredia.

Coll, M., Fonseca, C. \& Cortés, J. (2001). El manglar y otras asociaciones vegetales de la Laguna de Gandoca, Limón, Costa Rica. Rev. Biol. Trop., 49 (2), 321-329.

Coll, M., Cortés, J. \& Sauma, D. (2004). Características físico-químicas y determinación de plaguicidas en el agua de la Laguna de Gandoca, Limón, Costa Rica. Rev. Biol. Trop., 52 (2), 33-42.

Cotto, A. (2001). Guía de identificación de los peces marinos del Mar Caribe de Nicaragua. DIPAL II. Convenio Países Bajos-Nicaragua \# NI-007604. Nicaragua: Producciones EMCOR, S.A.

Dyer, K. R. (1979). Estuaries and estuarine sedimentation. En K. R. Dyer (Ed.), Estuarine Hydrography and Sedimentation (pp. 1-18). Cambridge, London: Editorial of Cambridge, University.

Fonseca, M. \& Solís, V. (2004). Gobernabilidad en el manejo de áreas silvestres protegidas en Costa Rica. La experiencia de manejo conjunto del Refugio Nacional de Vida Silvestre Gandoca-Manzanillo. Coope Sol I Dar, San José, Costa Rica: Editorial San José, Coope Sol I Dar R.L.

García, C., Claro, R. \& Koshelev, V. (1994). Reproducción, ecología de los peces marinos de Cuba. Instituto de Oceanografía Academia de Ciencias de Cuba. Centro de Investigaciones Quintana Roo. México: FERRANDIZ, S.A.

Gastézzi, A. P. (2003). Evaluación y manejo de los recursos de la Laguna de Gandoca-Manzanillo Costa Rica. Te- sis de Maestría no publicada. Regional en Manejo y Conservación de Vida Silvestre. Heredia, Costa Rica.

Google Earth. (2008). Google Earth. Recuperado en julio 15, 2008, disponible en http://www.google.es/

Green, Y. A. (1993). Ictioplancton del canal de agua dulce, estero Huizache-Caimanero. Tesis de Maestría no publicada. Centro Interdisciplinario de Ciencias Marinas, dependiente del Instituto Politécnico Nacional. La Paz, México.

Grimes, C. B. (1987). Reproductive biology of the Lutjanidae: a review. En J. J. Polovina \& S. Ralston (Eds.), Tropical snappers and grupers: biology and fisheries management (pp. 239-294). London: West. Press/Boulder.

Harbeyam, K. A., Horn, S. P. \& Umaña, G. (2003). Basic limnology of fifty-one lakes in Costa Rica. Rev. Biol. Trop., 51 (1), 107-122.

Howells, R. G. A. \& Sunski, J. (1990). Lower temperature tolerance of snook, Centropomus undecimalis. North. Gulf Sci., 11, 155-158.

Instituto Meteorológico Nacional. (2007). Índices de precipitación mensual en la Estación Meteorológica de Limón, Costa Rica. Gobierno de Costa Rica.

Marcano, L. (1990). Evaluación de la pesquería de arrastre en áreas de Margarita y norte de Sucre. Informe Técnico Estación Experimental Sucre (FONAIAP). Venezuela.

Pérez, M. (1999). Biología pesquera y aspectos ecológicos de la ictiofauna más importante de la cuenca de Laguna de Perlas en la Región Autónoma del Atlántico Sur (RASS) de Nicaragua. Proyecto para el Desarrollo Integral de la Pesca Artesanal en la Región Autó- 
noma Atlántico Sur (DIPAL II). Bluefields, Nicaragua.

Perry, J. A. \& Perry, S. D. (1974). Los peces comunes de la costa atlántica de Costa Rica. Ciudad Universitaria Rodrigo Facio. San José, Costa Rica: Editorial UCR.

Pickard, G. L. (1990). Descriptive Physical Oceanography. An Introduction. London: Pergamon, Oxford.

PREPAC. (2006). Informe Nacional sobre el Inventario de Cuerpos de Agua Continentales de Costa Rica. Plan Regional de Pesca y Acuicultura Continental (PREPAC). San Salvador, El Salvador.

Quiroga, B. C., Solís, C. F. \& Estrada, J. G. (1996). La pesquería de róbalo en México. Pesquerías Relevantes de México. XXX Aniversario del INP. SEMARNAP/INP (II): 559-578.

Ruiz, R. S., Lucano, R. G. \& Mariscal, R. J. (1997). Length-Weight relationships of soft-bottom demersal fishes from Jalisco and Colima states, México. Naga, ICLARM Quarterly, 20 (3/4), 62-63.

Windevoxhel, N., Villegas, F. \& Chacón, D. (1995). Humedal Gandoca. Manza- nillo: Ficha Técnica. REGAMA.FIC. ANAI-HUMEDALES. Costa Rica.

Yáñez-Arancibia, A. (1977). Taxonomía, ecología y estructura de las comunidades de peces en lagunas costeras con bocas efímeras del pacífico de México. En 39 th Annual Meeting American Society of Limnology and Oceanography. Savannah, Georgia.

Yáñez-Arancibia, A. (1986). Lagunas costeras y estuarios como ecosistemas. En A. Yáñez-Arancibia (AGT Ed.), Ecología de la zona costera (pp. 1416). México, D.F.: Editorial AGT.

Zarza, M. A., Berruecos, V. M., Vásquez, P. C. \& Álvarez, T. P. (2006). Cultivo experimental de róbalo Centropomus undecimalis (Bloch, 1792) y Chucumite Centropomus parallelus (Poey, 1860) (Perciformes: Centropomidae) en agua dulce en un estanque de concreto en Alvarado Veracruz, México. Rev. Veter. Mexico, 37 (3), 327-333.

Zúñiga, R. G. (2005). Guía de información turística (Catálogo) de la fauna ictiológica en la Laguna de Gandoca-Manzanillo. Práctica de Campo. Universidad Nacional, Heredia. 